\title{
Safety of elvitegravir, cobicistat, emtricitabine, and tenofovir alafenamide in HIV-1-infected adults with end-stage renal disease on chronic haemodialysis: an open-label, single-arm, multicentre, phase $3 b$ trial
}

Joseph J Eron Jr, Jean-Daniel Lelievre, Robert Kalayjian, Jihad Slim, Anson K Wurapa, Jeffrey L Stephens, Cheryl McDonald, Eric Cua, Aimee Wilkin, Brigitte Schmied, Mehri McKellar, Stephanie Cox, Sophia R Majeed, Shuping Jiang, Andrew Cheng, Moupali Das, Devi SenGupta

\begin{abstract}
Summary
Background Current treatment for HIV-infected individuals with renal failure on haemodialysis frequently requires complex regimens with multiple pills. A daily single-tablet regimen of coformulated elvitegravir, cobicistat, emtricitabine, and tenofovir alafenamide is approved in Europe, the USA, and in other regions for use in HIV-1-infected individuals with mild-to-moderate chronic kidney disease (creatinine clearance $30-69 \mathrm{~mL} / \mathrm{min}$ ). We aimed to assess the safety, efficacy, an $\mathrm{d}$ ph armacokinetics of th is re gimen in $\mathrm{HI}$ V-infected ad ults wi th en d-stage re nal di sease on ch ronic haemodialysis.
\end{abstract}

Methods We did an open-label, single-arm, multicentre, phase $3 b$ trial at 26 outpatient clinics in Austria, France, Germany, and the USA. Participants were HIV-1-infected adults with end-stage renal disease (creatinine clearance $<15 \mathrm{~mL} / \mathrm{min}$ ), on chronic haemodialysis for at least $\mathbf{6}$ months before screening. Virological suppression (ie, plasma HIV-1 RNA $<50$ copies per $\mathrm{mL}$ ) on a stable antiretroviral regimen was required for at least 6 months before screening with a CD4 count of at least 200 cells per $\mu \mathrm{L}$. We switched all participants to coformulated elvitegravir $150 \mathrm{mg}$, cobicistat $150 \mathrm{mg}$, emtricitabine $200 \mathrm{mg}$, and tenofovir alafenamide $10 \mathrm{mg}$ once daily, taken after haemodialysis for up to 96 weeks. We did assessments at study visits at weeks $2,4,8,12,24,36$, and 48 , and every 12 weeks thereafter up to 96 weeks. The primary endpoint was the incidence of treatment-emergent adverse events of grade 3 or higher up to week 48 . All participants who received at least one dose of study drug were included in the primary analysis. This study is registered with ClinicalTrials.gov (NCT02600819) and is closed to new participants.

Findings Between Feb 1, and Nov 3, 2016, 55 participants were enrolled and received at least one dose of study drug Through week 48, 18 of 55 participants (33\%, 95\% CI 20-45) had an adverse event of grade 3 or higher on study treatment. Treatment-emergent grade 3 or higher adverse events that occurred in more than one participant included anaemia, osteomyelitis, prolonged electrocardiogram QT, fluid overload, hyperkalaemia, hypertension, and hypotension (all $n=2$ ). No adverse event of grade 3 or higher was considered by the site investigators to be treatment related. Three participants (5\%, 95\% CI 0-11) discontinued treatment because of adverse events; one of these (grade 1 allergic pruritus) was considered treatment related. Treatment-related adverse events were reported for six individuals $\mathbf{( 1 1 \%}$ 95\% CI 3-19), the most common of which was nausea (in four individuals [7\%]); all treatment-related adverse events were grade 1 or 2 in severity.

Interpretation At 48 weeks, switching to the single-tablet regimen of elvitegravir, cobicistat, emtricitabine, and tenofovir alafenamide was well tolerated. This regimen might provide a tolerable and convenient option for ongoing treatment of HIV-1 infection in adults with end-stage renal disease on chronic haemodialysis.

Funding Gilead Sciences.

\section{Introduction}

Currently available antiretroviral drugs are highly effective and have thus led to striking improvements in survival and disease progression in HIV-1-infected individuals. The paradigm of HIV treatment has shifted to that of treating HIV-infected individuals with longterm, chronic illness in an increasingly aging population who are at risk for non-AIDS-associated comorbidities, such as those of the kidney, bone, and liver, and cardiac disease. ${ }^{1-3}$ Previous analyses ${ }^{4,5}$ that included an age-matched, control cohort noted that end-stage renal disease occurred at a younger age in HIV-1-infected individuals than in HIV-negative controls. ${ }^{4,5}$ Given the prevalence of end-stage renal disease in people with HIV and the likelihood that its prevalence will increase with the aging of the population and the common comorbidities of hypertension and diabetes, a safe and convenient antiretroviral regimen
Lancet HIV 2019; 6: e15-24

Published Online

December 13, 2018

http://dx.doi.org/10.1016/

S2352-3018(18)30296-0

See Comment page e2

Department of Medicine, Division of Infectious Diseases, UNC School of Medicine, Chapel Hill, NC, USA

(Prof JJ Eron Jr MD); Department of Clinical Immunology \& Infectious Diseases, Hôpital Henri Mondor, Créteil, France (ProfJ-D Lelievre MD); Infectious Diseases, MetroHealth, Cleveland, $\mathrm{OH}$, USA (R Kalayjian MD); Infectious Diseases, Saint Michael's Medical Center, Newark, N USA (J Slim MD); Infectious Disease Specialists of Atlanta PC, Decatur, GA, USA (A K Wurapa MD); Department of Internal Medicine, Mercer University School of Medicine, Macon, GA, USA (Prof J L Stephens MD); Tarrant County Infectious Disease Associates, Fort Worth, TX, USA (C McDonald MD); Department of Infectious Diseases, Hôpital I'Archet, Centre Hospitalier Universitaire de Nice, Nice, France (E Cua MD); Section on Infectious Disease, Wake Forest University School of Medicine, Winston-Salem, NC, USA (A Wilkin MD); Sozialmedizinisches Zentrum Baumgartner Höhe Otto-Wagner Hospital, Vienna Austria (B Schmied MD); Infectious Diseases, Department of Medicine, Duke University School of Medicine, Durham, NC, USA (M McKellar MD); and Department of Virology (S Cox BS), Department of Clinical Pharmacology (S R Majeed PharmD), Department of Biometrics ( $\mathrm{S}$ jiang $\mathrm{PhD}$ ), and Department of HIV Clinical Research, 


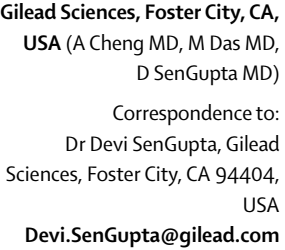

Devi.SenGupta@gilead.com

Research in context

\section{Evidence before this study}

We searched PubMed for published clinical trials of tenofovir alafenamide in patients with HIV-1-infection and renal disease, with the title or abstract search terms "tenofovir alafenamide" and "HIV" and "study" or "trial" and "renal disease" or "renal impairment." Searches were limited to articles published in English between Jan 1, 1997, and March 1, 2018. Our search yielded three articles, one of which we excluded because it summarised the pharmacokinetics of tenofovir alafenamide in individuals without HIV infection and with severe renal impairment. The remaining two summarised results at weeks 48 and 96, respectively, from an open-label, single-arm phase 3 study assessing coformulated elvitegravir, cobicistat, emtricitabine, and tenofovir alafenamide in HIV-infected adults with a creatinine clearance of $30-69 \mathrm{~mL} / \mathrm{min}$. The treatment maintained virological suppression and was well tolerated in these individuals with mild-to-moderate renal impairment.

is needed for patients with HIV on chronic haemodialysis. However, although kidney transplantation has been shown to confer a survival advantage compared with remaining on dialysis in HIV-infected individuals, there remain substantial disparities in access to transplantations among black individuals living with HIV in the USA, particularly in the south-eastern area, and also among ethnic minorities and less-advantaged indigenous populations in developed countries. ${ }^{7.8}$

On top of an increased risk of age-related comorbidities, individuals with HIV are at risk for acute and chronic kidney disease from a wide spectrum of other causes including HIV-associated nephropathy, immune complex kidney disease, thrombotic microangiopathy, and kidney disease associated with comorbidities such as diabetes, hypertension, hepatitis B and hepatitis C coinfections, and use of medications associated with nephrotoxicity. ${ }^{9,10}$ The prevalence of HIV-1 infection in patients with endstage renal disease is an estimated $0.5 \%$ in Europe ${ }^{11}$ and $1.5 \%$ in the USA, ${ }^{12}$ although this might be an underestimate of current rates ${ }^{13}$ since more recent values are not available. Furthermore, the prevalence and incidence of chronic kidney disease and end-stage renal disease are expected to rise as life expectancy improves for people living with HIV. ${ }^{14,15} \mathrm{New}$ antiretroviral therapies (ARTs) that advance the current standard of care are needed to help health-care providers better manage comorbidities such as renal disease, since their HIV-1-infected patients expect to receive life-long treatment.

Current guidelines recommend initial treatment of HIV with a combination of two nucleoside reverse transcriptase inhibitors (NRTIs) with a drug from another class..$^{16-18}$ However, most NRTIs are renally eliminated ${ }^{19}$ which raises concerns for the use of this drug class in a population with impaired kidney

\section{Added value of this study}

This is the first study to assess switching to a single-tablet regimen of elvitegravir, cobicistat, emtricitabine, and tenofovir alafenamide in HIV-infected adults with end-stage renal disease on chronic haemodialysis who had been previously maintained on a stable antiviral regimen for at least 6 months. The results suggested that the single-tablet regimen was well tolerated in this patient population through 48 weeks, with maintenance of virological suppression and increased patient satisfaction.

\section{Implications of all the available evidence}

Convenient, well tolerated treatment options are scarce for the growing demographic of HIV-1-infected individuals who have an unmet medical need for safer, more convenient antiretroviral regimens. The single-tablet regimen of elvitegravir, cobicistat, emtricitabine, and tenofovir alafenamide might provide a safe and convenient option for ongoing treatment of HIV-1 infection in adults with end-stage renal disease on haemodialysis.

function. As such, ART options for HIV-infected individuals with chronic kidney disease are few because the long-term use of tenofovir disoproxil fumarate has been associated with nephrotoxicity ${ }^{20}$ and abacavir has been associated with increased cardiovascular risk. ${ }^{21}$ For individuals who cannot be treated with either of these drugs, alternative nucleoside-sparing regimens have been suggested in treatment guidelines, ${ }^{16-18}$ however, this approach is hampered because of concerns of reduced virological activity, drug-drug interactions, or tolerability of these combinations. ${ }^{16-18}$ Additional challenges associated with currently available ART regimens in this population also exist, including regimen complexity, pill burden (if more than one is needed), and risk of dosing errors. ${ }^{22}$

The NRTI tenofovir alafenamide, a novel prodrug of tenofovir, achieves similar antiretroviral efficacy at a lower dose than tenofovir disoproxil fumarate, resulting in $91 \%$ lower plasma tenofovir exposures in individuals with normal renal function. ${ }^{23}$ Several large, phase 3 studies of HIV-1-infected, treatment-naive and virologically suppressed individuals, including those with mild-to-moderate renal impairment, have shown longterm safety and favourable renal and bone profiles with tenofovir alafenamide-containing regimens compared with those containing tenofovir disoproxil fumarate. ${ }^{24-26}$ The single-tablet regimen containing elvitegravir, cobicistat, emtricitabine, and tenofovir alafenamide is one of the recommended initial regimens in HIV-1 treatment guidelines in the USA and Europe. ${ }^{16-18}$ This regimen can be given without dose adjustment to individuals with a creatinine clearance as low as $30 \mathrm{~mL} / \mathrm{min}^{27}$

In this study, we assessed the safety and efficacy of switching to a single-tablet regimen of coformulated 
elvitegravir, cobicistat, emtricitabine, and tenofovir alafenamide once daily in HIV-1-infected, virologically suppressed adults with end-stage renal disease who were on chronic haemodialysis at study entry. Here we present the results at 48 weeks.

\section{Methods \\ Study design and participants}

We did a multicentre, open-label, single-arm, phase 3b trial (GS-US-292-1825) at 26 outpatient clinics in Austria, France, Germany, and the USA. This study was approved by central or site-specific $r$ eview b oards or ethics committees.

Study investigators enrolled HIV-1-infected adults (aged at least 18 years) with end-stage renal disease (creatinine clearance $<15 \mathrm{~mL} / \mathrm{min}$ ) on chronic haemodialysis for at least 6 months before screening. Participants were required to be virologically suppressed (ie, plasma HIV-1 RNA $<50$ copies per $\mathrm{mL}$ ) on a stable antiretroviral regimen for at least 6 consecutive months before screening, with a CD4 count of at least 200 cells per $\mu \mathrm{L}$ and no documented history of HIV resistance to elvitegravir, emtricitabine, lamivudine, or tenofovir. All participants provided written informed consent. A subset of participants provided additional written, informed consent for a pharmacokinetic substudy.

\section{Procedures}

Eligible participants received a single tablet once daily of coformulated elvitegravir $150 \mathrm{mg}$, cobicistat $150 \mathrm{mg}$, emtricitabine $200 \mathrm{mg}$, and tenofovir alafenamide $10 \mathrm{mg}$, taken after haemodialysis on dialysis days, for up to 96 weeks. We undertook post-baseline study visits at weeks $2,4,8,12,24,36$, and 48, and every 12 weeks thereafter (up to 96 weeks). Safety was assessed by physical examinations, laboratory tests, 12-lead electrocardiogram, use of concomitant drugs, and recording of adverse events, which were coded using the Medical Dictionary for Regulatory Activities (MedDRA, version 20.0). Laboratory tests included haematological analysis, serum chemistry tests, fasting lipid parameters, CD4 cell counts, and serum creatinine concentrations to measure creatinine clearance (estimated glomerular filtration rate by Cockcroft Gault; Covance Laboratories, Indianapolis, IN, USA). Blood samples for chemistry tests, including serum creatinine, were drawn before haemodialysis. Laboratory tests also included measurement of plasma HIV-1 RNA (Roche TaqMan 2.0; Roche Diagnostics, Rotkreuz, Switzerland). Protocol-defined resistance testing consisted of genotypical and phenotypical analysis of integrase, protease, and reverse transcriptase (Monogram Biosciences, South San Francisco, CA, USA) for any participant who had a confirmed plasma HIV-1 RNA of at least 50 copies per $\mathrm{mL}$ with the confirmation p lasma H IV-1 R NA a t 1 east 200 c opies per $\mathrm{mL}$ or who had a plasma HIV-1 RNA of at least 200 copies per $\mathrm{mL}$ in the week 48 window (between days 295 and 378 [inclusive] of the study), or at the last visit on study drug.

The pharmacokinetics of elvitegravir, cobicistat, emtricitabine, tenofovir alafenamide, and its active metabolite, tenofovir, were assessed in a dedicated, intensive pharmacokinetic substudy. The substudy was done at the week 2 or 4 visit after an observed dose on a day before haemodialysis and when participants had been given three consecutive doses of the single-tablet study drug between two haemodialysis sessions to assess plasma concentrations of the study-drug components at their highest expected concentrations. A trough pharmacokinetic blood sample was obtained before administration of an observed dose and intensive pharmacokinetic blood sampling was done post dose at $0 \cdot 5,1,2,3,4,6,8$, and $24 \mathrm{~h}$. Plasma concentrations of elvitegravir, cobicistat, emtricitabine, tenofovir alafenamide, and tenofovir were determined using fully validated, high-performance liquid chromatography tandem-mass spectroscopy bioanalytical methods.

We measured patient-reported outcomes (ie, HIV treatment satisfaction questionnaires [HIV-TSQ], comprising the status and change versions) at week 24 and every 24 weeks thereafter. The HIV-TSQ was administered at screening, day 1 , and week 4 . Medical adherence assessments were done on day 1 and at every post-baseline visit. All patient-reported outcome assessments were done at the early study drug discontinuation visit.

\section{Outcomes}

The primary outcome was the incidence of treatmentemergent adverse events of grade 3 or higher up to week 48. A treatment-emergent grade 3 or higher adverse event was defined as a grade 3 or higher adverse event with either an onset date on or after the studydrug start date and no later than 30 days after permanent discontinuation of the study drug or one that led to premature discontinuation of study drug. Secondary outcomes were the incidence of treatment-emergent grade 3 or higher adverse events up to week 96, the proportion of participants who had plasma HIV-1 RNA less than 50 copies per $\mathrm{mL}$ at weeks 24, 48, and 96 as defined by the US Food and Drug Administration (FDA) snapshot algorithm ${ }^{28}$ for the overall population and by subgroups of age, sex, race, geographical region, and study-drug adherence rate, the proportion of participants who had plasma HIV-1 RNA less than 20 copies per $\mathrm{mL}$ (snapshot algorithm), the proportion of participants with plasma HIV-1 RNA less than 50 copies per $\mathrm{mL}$ at week 48 when imputing missing as failure and missing as excluded, and the absolute change in CD4 cell count and percentage from baseline at week 48. Patient-reported outcomes and steady-state pharmacokinetic parameters of elvitegravir, cobicistat, emtricitabine, tenofovir alafenamide, and tenofovir were also determined as secondary outcomes. All week 96 outcomes will be reported separately. 


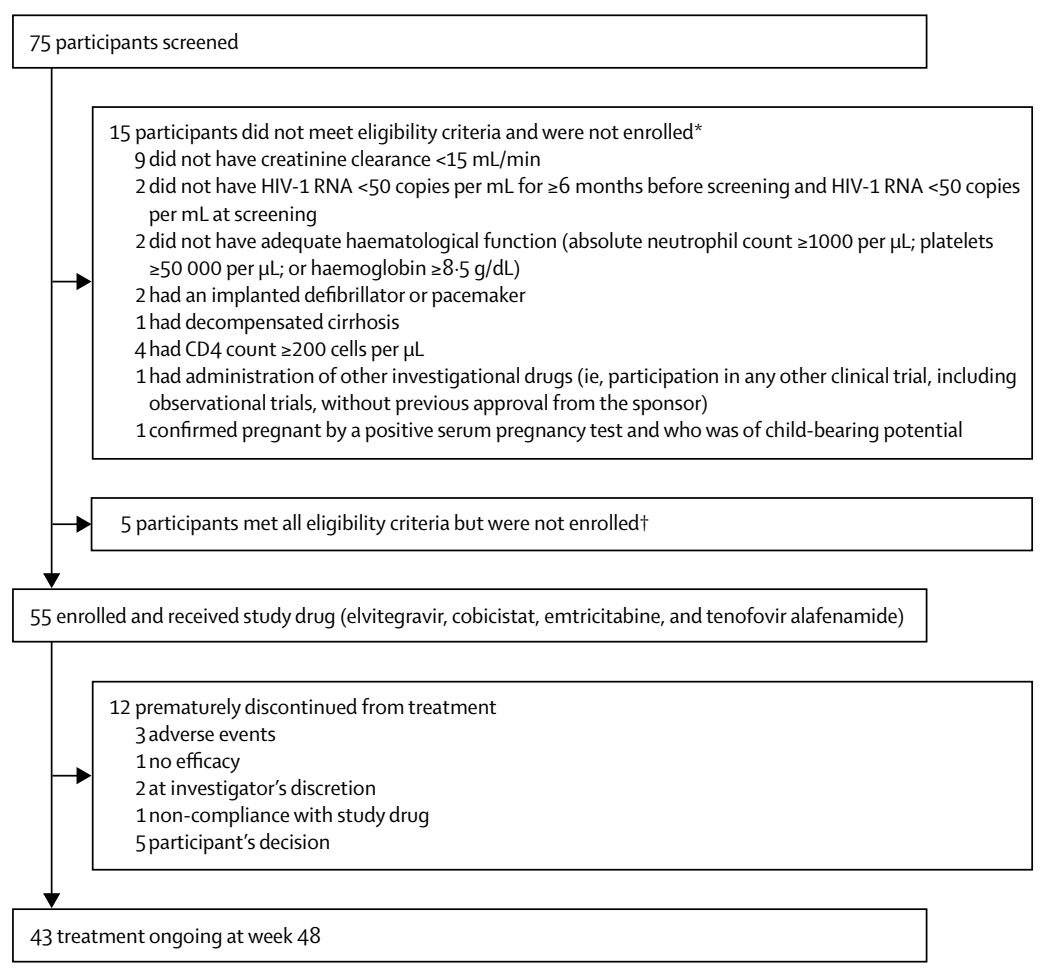

Figure: Trial profile

*Screen failure participants who did not meet the study eligibility criteria might have had more than one criterion that led to ineligibility. †Among the five participants who met all eligibility criteria but were not enrolled, four withdrew consent and one was outside the visit window for their baseline visit.

\section{Statistical analysis}

We did the primary analysis after all enrolled participants had completed their week 48 study visit or had prematurely discontinued the study drug. All participants who were enrolled in the study and received at least one dose of study drug (safety analysis set) were included in the primary analysis. One planned independent data monitoring committee interim analysis was done after the first 25 participants enrolled had completed their week 12 visit or prematurely discontinued the study drug. The independent data monitoring committee concluded that efficacy and safety findings warranted continuation of the trial. For analysis of the primary study endpoint, grade 3 or higher adverse events up to the week 48 visit for each participant were included. For those who discontinued study drug before the week 48 visit, grade 3 or higher adverse events up to the last dose date plus 30 days were included. The derived $95 \% \mathrm{CI}$ of the primary endpoint was based on normal approximation. A planned sample size of 50 participants was based on feasibility and considered sufficient to ensure a positive lower bound of the primary objective of the study. In a previous study, ${ }^{26}$ the grade 3 or 4 adverse event rate among virologically suppressed adults with mild-to-moderate renal impairment (creatinine clearance $<50 \mathrm{~mL} / \mathrm{min}$ ) who switched to elvitegravir, cobicistat, emtricitabine, and tenofovir alafenamide was $8.8 \%$ at week 48 . With an assumed rate of grade 3 or higher adverse events of $10 \%$, 50 participants provided $95 \%$ confidence for the primary endpoint to be between $1.7 \%$ and $18 \cdot 3 \%$, assuming normal approximation to binomial proportions. Other safety data were described using all data collected on or after study drug was first given up to either the data cutoff date or, for participants who discontinued treatment early, up to 30 days after the last dose of study drug.

In the snapshot analysis, participants who were enrolled in the study and received at least one dose of study drug (full analysis set) were classified in the following three outcome groups based on plasma HIV-1 RNA collected at week 48 while on study treatment: 1) plasma HIV-1 RNA less than 50 copies per $\mathrm{mL}$ at week 48 (between days 295 and 378, inclusive); 2) plasma HIV-1 RNA at least 50 copies per $\mathrm{mL}$, including the following three types of participants: plasma HIV-1 RNA at least 50 copies per $\mathrm{mL}$ at week 48; participants who discontinued study drug due to reasons other than absence of efficacy before or in week 48 with last plasma HIV-1 RNA at least 50 copies per $\mathrm{mL}$; and participants who discontinued study drug before or in week 48 because of an absence of efficacy; and 3) no virological data in the week 48 window, including for the following two types of participants: those who discontinued study drug for reasons other than absence of efficacy before or in week 48 with last available plasma HIV-1 RNA less than 50 copies per $\mathrm{mL}$, and participants who were still on study drug with missing plasma HIV-1 RNA data at week 48 . The week 48 efficacy endpoint was also analysed by snapshot analysis with a plasma HIV-1 RNA cutoff of 20 copies per $\mathrm{mL}$, and by analysis of the proportion of participants with plasma HIV-1 RNA less than 50 copies per $\mathrm{mL}$ through imputing the category of no virological data as a failure (missing=failure) and as excluded (missing=excluded).

Patient-reported outcomes were measured in the safety analysis set using the HIV-TSQ, SF-36 (which assesses general quality-of life measures), and medication adherence questionnaires. For the medication adherence questionnaire, on-treatment data collected up to 1 day plus the last dose date of study drug were included in the summary. For all other questionnaires, data collected up to 30 days plus the last dose date of study drug were included in summaries. Multiple responses and out-ofrange responses were set to missing and missing responses were not imputed. Study drug adherence was computed as the number of pills taken divided by the number of pills prescribed (where the number of pills taken was the number of pills dispensed minus the number of pills returned). We used SAS version 9.4 for all analyses. Pharmacokinetic parameters were calculated by application of a nonlinear model using standard noncompartmental analysis (Phoenix WinNonlin version 6.4 
[Certara USA, Princeton, NJ, USA]). This study is registered with ClinicalTrials.gov, number NCT02600819.

\section{Role of the funding source}

The funder of the study had the lead role in study design, data collection, data analysis, data interpretation, and (along with JJEJ) writing of the manuscript. The corresponding author had full access to all the data in the study and had final responsibility for the decision to submit for publication.

\section{Results}

Between Dec 15, 2015, and Nov 3, 2016, we screened 75 participants, and between Feb 1, and Nov 3, 2016, 55 were enrolled and received at least one dose of study drug (figure). M ost participants w ere $m$ ale a nd b lack, with a median age of 51 years (table 1). Baseline characteristics also reflected a $h$ igh $p$ rominence of comorbidities (eg, diabetes, hypertension, cardiovascular disease, and hyperlipidaemia).

Treatment-emergent grade 3 or higher adverse events at week 48 were reported for 18 of 55 participants (33\%, $95 \%$ CI 20-45; table 2, appendix pp 2-12). No grade 3 or higher adverse event was considered by the site investigator to be treatment related. Treatment-emergent grade 3 or higher adverse events that occurred in more than one participant included anaemia, osteomyelitis, prolonged electrocardiogram QT, fluid overload, hyperkalaemia, hypertension, and hypotension (all $n=2$ ). Although not considered to be treatment related, three events (grade 4 generalised oedema, grade 3 prescheduled renal transplant, and grade 1 pruritus allergic) led to premature discontinuation of study drug.

Overall, treatment with elvitegravir, cobicistat, emtricitabine, and tenofovir alafenamide was well tolerated, with most adverse events reported as mild or moderate in severity. Adverse events leading to study drug discontinuation were uncommon, occurring in three of 55 participants (5\%, 95\% CI 0-11) and included grade 4 generalised oedema $(n=1)$, grade 3 scheduled renal transplant $(n=1)$, and grade 1 allergic pruritus $(n=1)$; only the pruritus event was considered to be related to study drug. Treatment-related adverse events were reported for six individuals $(11 \%$, 95\% CI 3-19 which included nausea [ $n=4]$ ), and diarrhoea, dyspepsia, asthenia, myalgia, polyuria, and allergic pruritus ( $n=1$ each). One individual died from heart failure and anasarca following staphylococcal endocarditis, events that were not considered related to study drug.

For adverse events potentially associated with emtricitabine based on previous studies (ie, among those listed in the prescribing information as having an incidence of at least $10 \%$ ) regardless of investigator attribution, the most commonly reported were grade 1 or 2 nausea, cough, and diarrhoea. Adverse events in this category considered related to study drug were reported for five participants (9\%); most related events

\begin{tabular}{|c|c|c|}
\hline & $\begin{array}{l}\text { Elvitegravir, cobicistat, } \\
\text { emtricitabine, } \\
\text { and tenofovir } \\
\text { alafenamide }(n=55)\end{array}$ & \\
\hline Age (years; median [range]) & $51(23-64)$ & \\
\hline \multicolumn{3}{|l|}{ Sex at birth } \\
\hline Male & $42(76 \%)$ & \\
\hline Female & $13(24 \%)$ & \\
\hline \multicolumn{3}{|l|}{ Race } \\
\hline Black & $45(82 \%)$ & \\
\hline White & $10(18 \%)$ & \\
\hline Body-mass index (kg per m²) & $26 \cdot 3(23 \cdot 5-30 \cdot 4)$ & \\
\hline Creatinine clearance $(\mathrm{mL} / \mathrm{min})$ & $10 \cdot 9(8 \cdot 8-13 \cdot 8)$ & \\
\hline Duration of haemodialysis (years) & $6(4-10)$ & \\
\hline HIV-1 RNA < 50 copies per $\mathrm{mL}$ & $54(98 \%)$ & \\
\hline CD4 count (cells per $\mu \mathrm{L}$ ) & $515(387-672)$ & \\
\hline$<500$ & $26(47 \%)$ & \\
\hline$\geq 500$ & $29(53 \%)$ & \\
\hline \multicolumn{3}{|l|}{ HIV disease status } \\
\hline Asymptomatic & $42(78 \%)$ & \\
\hline Symptomatic HIV infection & $2(4 \%)$ & \\
\hline AIDS & $10(19 \%)$ & \\
\hline \multicolumn{3}{|l|}{ Mode of infection } \\
\hline Heterosexual sex & $33(60 \%)$ & \\
\hline Men who have sex with men & $15(27 \%)$ & \\
\hline Intravenous drug use & $3(5 \%)$ & \\
\hline Blood transfusion & $3(5 \%)$ & \\
\hline Vertical transmission & $3(5 \%)$ & \\
\hline Other & $1(2 \%)$ & \\
\hline Unknown & $3(5 \%)$ & \\
\hline \multicolumn{3}{|l|}{ Medication used before antiretroviral switch } \\
\hline Containing tenofovir disoproxil fumarate & $16(29 \%)$ & \\
\hline Containing abacavir & $31(56 \%)$ & \\
\hline $\begin{array}{l}\text { Containing neither tenofovir disoproxil } \\
\text { fumarate or abacavir }\end{array}$ & $10(18 \%)$ & \\
\hline Positive for hepatitis C virus antibodies & $12(22 \%)$ & \\
\hline \multicolumn{3}{|l|}{ Clinical history } \\
\hline Diabetes & $15(27 \%)$ & \\
\hline Hypertension & $52(95 \%)$ & \\
\hline Cardiovascular disease & $26(47 \%)$ & \\
\hline Hyperlipidaemia & $23(42 \%)$ & \\
\hline
\end{tabular}

Data are $\mathrm{n}(\%)$ or median (IQR) except where noted otherwise.

Table 1: Baseline demographic and clinical characteristics

were grade 1 or 2 . None of these events led to premature discontinuation of study drug. The most common event, occurring in four of these five participants, was grade 1 or 2 nausea.

No clinically relevant changes occurred from baseline in median values for haematology or clinical chemistry parameters. Creatinine clearance remained stable throughout the study, with a median (IQR) change from baseline at week 48 of $0.1 \mathrm{~mL} / \mathrm{min}(-1.9$ to 1.8$)$. Overall, 24 participants $(44 \%)$ had grade 3 or 4 laboratory abnormalities, the most common of which 


\begin{tabular}{|c|c|}
\hline & $\begin{array}{l}\text { Elvitegravir, cobicistat, } \\
\text { emtricitabine, } \\
\text { and tenofovir } \\
\text { alafenamide }(n=55)\end{array}$ \\
\hline Grade 3 or higher adverse event by week 48 & $18(33 \%)$ \\
\hline Grade 3 adverse events & $14(25 \%)$ \\
\hline Grade 4 adverse events & $4(7 \%)$ \\
\hline Any adverse event & $51(93 \%)$ \\
\hline \multicolumn{2}{|l|}{ Adverse events noted in $\geq 10 \%$ of study population } \\
\hline Nausea & $12(22 \%)$ \\
\hline Hyperkalaemia & $8(15 \%)$ \\
\hline Pneumonia & $7(13 \%)$ \\
\hline Cough & $6(11 \%)$ \\
\hline Serious adverse events & $29(53 \%)$ \\
\hline Study drug-related adverse events* & $6(11 \%)$ \\
\hline Study drug-related serious adverse event & 0 \\
\hline $\begin{array}{l}\text { Adverse event leading to study drug } \\
\text { discontinuation } \dagger\end{array}$ & $3(5 \%)$ \\
\hline Death $\ddagger$ & $1(2 \%)$ \\
\hline $\begin{array}{l}\text { Any common adverse event associated with } \\
\text { emtricitabine } \$\end{array}$ & $25(45 \%)$ \\
\hline Grade 1 or 2 & $24(44 \%)$ \\
\hline Grade 3 & $1(2 \%) \boldsymbol{\top}$ \\
\hline Grade 4 & 0 \\
\hline \multicolumn{2}{|l|}{ Adverse events noted in $\geq 5 \%$ of study population } \\
\hline Nausea & $12(22 \%)$ \\
\hline Cough & $6(11 \%)$ \\
\hline Diarrhoea & $5(9 \%)$ \\
\hline Asthenia & $3(5 \%)$ \\
\hline Dizziness & $3(5 \%)$ \\
\hline Headache & $3(5 \%)$ \\
\hline
\end{tabular}

Data are $\mathrm{n}(\%)$. All adverse events up to data cut were included, except for the primary endpoint of grade 3 or 4 adverse events, which included adverse events up to the week 48 visit for each participant. *Most common study drug-related adverse event was nausea $(n=4 ; 7 \%)$. †Adverse events leading to study drug discontinuation included generalised oedema $(n=1)$, allergic pruritus $(n=1)$, and renal transplantation $(n=1)$. $\neq$ Cause of death was heart failure and anasarca following staphylococcal endocarditis $(n=1)$. \$Common adverse events $(\geq 10 \%)$ in emtricitabine prescribing information. ${ }^{28}$ Grade 3 dizziness.

Table 2: Adverse events

was increased amylase ( $\mathrm{n}=9$ [16\%] of 55; appendix $\mathrm{p} 13)$. As is characteristic for individuals with end-stage renal disease, median values greater than reference values occurred at baseline and post baseline for creatinine, blood urea nitrogen, phosphate, parathyroid hormone, and amylase; otherwise, median values were generally within the relevant reference ranges. Median (IQR) change in amylase from baseline at week 48 was $-2 \mathrm{U} / \mathrm{L}$ (-36 to 21). There were no clinically relevant changes from baseline in median fasting values for total cholesterol, direct LDL cholesterol, HDL cholesterol, total cholesterol to HDL ratio, triglycerides, or glucose in serum at week 48 (appendix p 14).

In the subset of individuals $(n=12)$ who participated in the intensive pharmacokinetic substudy, the measurements of plasma concentrations of elvitegravir, cobicistat, emtricitabine, tenofovir alafenamide, and tenofovir were taken when concentrations of the renally eliminated drug components would be expected to be highest (ie, on a day before haemodialysis, after three doses in between two dialysis sessions). Exposures of elvitegravir, cobicistat, and tenofovir alafenamide, which are metabolised through the liver, were consistent with the range of historical data in HIV-1 infected adults with normal renal function (table 3). ${ }^{27,29}$ As expected, given that tenofovir and emtricitabine are renally eliminated, mean exposures (area under the concentration curve [AUC]) of these analytes in individuals on haemodialysis were higher than historical data obtained following the administration of elvitegravir, cobicistat, emtricitabine, and tenofovir alafenamide to HIV-1-infected adults with normal renal function or with mild-to-moderate renal impairment (table 3, appendix p 16). In comparison, tenofovir and emtricitabine exposures $\left(\mathrm{AUC}_{0-48 \mathrm{~h}}\right.$ for tenofovir and $\mathrm{AUC}_{\mathrm{in}}$ for emtricitabine) following single-dose administration of tenofovir disoproxil fumarate $300 \mathrm{mg}$ or emtricitabine $200 \mathrm{mg}$ in adults on haemodialysis treated in previous studies were 44900 and $53200 \mathrm{ng} \cdot \mathrm{h} / \mathrm{mL}$, respectively (appendix p 16). Based on these data, these exposures are anticipated in individuals on haemodialysis dosed once weekly with tenofovir disoproxil fumarate, and once every 4 days with emtricitabine.

45 participants $(81.8 \%)$ had plasma HIV-1 RNA less than 50 copies per $\mathrm{mL}$ at week 48 by the snapshot algorithm (table 4). Of the remaining ten participants, one had plasma HIV-1 RNA of at least 50 copies per mL within the week 48 visit window and subsequently resupressed. Another was found after enrolment to have pre-existing resistance to emtricitabine (Met184Val) and elvitegravir (Gly140Ser, Gln148His) and developed treatment-emergent resistance to tenofovir (Lys65Arg) while on study. He was therefore discontinued from study drug because of absence of efficacy. This participant later resuppressed on dolutegravir plus cobicistatboosted darunavir. Of the other eight participants, seven had no virological data in the corresponding window because they had prematurely discontinued study drug for reasons other than efficacy, with their last HIV-1 RNA value less than 50 copies per $\mathrm{mL}$. Reasons for premature discontinuation included adverse events, loss to followup, non-compliance, and protocol violation. The last participant was missing data during the week 48 window, but was still on study drug and remained suppressed at subsequent timepoints. When data were analysed imputing the category of no virological data as excluded, nearly all participants had plasma HIV-1 RNA less than 50 copies per $\mathrm{mL}$ at week 48 (table 4 ).

Results from the missing=failure analysis were consistent with the snapshot analysis (table 4). Efficacy was similar among subgroups (appendix p 15). Using the lower plasma HIV-1 RNA threshold of less than 20 copies per $\mathrm{mL}$, the proportion of participants meeting this criterion at week 48 by the snapshot algorithm was also 
81.8\%. CD4 cell counts and CD4 percentages remained stable through week 48 . The median (IQR) change in CD4 count from baseline at week 48 was -17 cells per $\mu \mathrm{L}$ $(-108$ to 88$)$, and the median (IQR) change in CD4 percentage from baseline at week 48 was $1 \cdot 4 \%(0 \cdot 9-4 \cdot 3)$. As measured with the HIV-TSQ at week $48,78 \%$ of participants felt much more satisfied overall with elvitegravir, cobicistat, emtricitabine, and tenofovir alafenamide compared with their pre-switch baseline regimens and $82 \%$ were much more satisfied with the convenience of taking a single-tablet regimen (appendix pp 17-37). Mean adherence at all post-baseline visits through week 48 was more than $93 \%$ as measured using a visual analogue score and more than $96 \%$ as measured by pill count (appendix pp 38-39).

\section{Discussion}

At 48 weeks, switching to the single-tablet regimen of coformulated elvitegravir, cobicistat, emtricitabine, and tenofovir alafenamide in HIV-1-infected adults with end-stage renal disease on chronic haemodialysis was well tolerated, as evidenced by an overall low incidence of adverse events that were considered to be treatment related or that led to early discontinuation of study drug, with maintenance of virological suppression. This was the first clinical trial to assess the safety and efficacy of switching to a daily single-tablet regimen in this patient population. The frequency of treatmentemergent grade 3 or higher adverse events in our study up to week 48 (the primary endpoint) was substantially higher than would be expected in a study of otherwise healthy adults living with HIV. However, our results were not unexpected in a population of HIV-infected adults on dialysis for a median of 6 years, nearly all of whom had hypertension, approximately half had cardiovascular disease or hyperlipidaemia, and a quarter had diabetes.

We detected no pattern to the actual distribution and types of treatment-emergent grade 3 or higher adverse events that were reported, because no event occurred in more than two participants. Indeed, the grade 3 or higher adverse events were primarily driven by complications or related to the above pre-existing medical conditions. We found it encouraging that none of the grade 3 or higher adverse events in our study were considered treatment related, though we acknowledge that there might be ascertainment bias in an open-label study. We note that our assumed $10 \%$ rate of grade 3 or higher adverse events was based on a previous study in adults with mild-tomoderate renal impairment, who were generally in better health at baseline than the individuals our current study. Therefore, it is reasonable to observe a higher than assumed grade 3 or higher adverse event rate in our current study.

Not unexpectedly, given the medically complex study population with underlying end-stage renal disease, most participants had at least one adverse event overall,

\begin{tabular}{|c|c|c|c|c|}
\hline & \multicolumn{2}{|c|}{ End-stage renal disease } & \multicolumn{2}{|c|}{ Healthy renal function } \\
\hline & $\mathrm{N}$ & $\begin{array}{l}\text { Mean (percentage } \\
\text { coefficient of } \\
\text { variation) }\end{array}$ & $\mathrm{N}$ & $\begin{array}{l}\text { Mean (percentage } \\
\text { coefficient of } \\
\text { variation) }\end{array}$ \\
\hline \multicolumn{5}{|l|}{ Elvitegravir } \\
\hline$A \cup C_{\text {tau }}(h \cdot n g / m L)$ & 10 & $14300(55 \%)$ & 19 & $22800(35 \%)$ \\
\hline$C_{\max }(\mathrm{ng} / \mathrm{mL})$ & 12 & $1260(55 \%)$ & 19 & $2110(34 \%)$ \\
\hline $\mathrm{C}_{\text {tau }}(\mathrm{ng} / \mathrm{mL})$ & 10 & $174(60 \%)$ & 19 & $287(62 \%)$ \\
\hline \multicolumn{5}{|l|}{ Cobicistat } \\
\hline$A \cup C_{\text {tau }}(h \cdot n g / m L)$ & 11 & $10200(59 \%)$ & 19 & $9460(34 \%)$ \\
\hline $\mathrm{C}_{\max }(\mathrm{ng} / \mathrm{mL})$ & 12 & $1370(67 \%)$ & 19 & $1450(28 \%)$ \\
\hline $\mathrm{C}_{\mathrm{tau}}(\mathrm{ng} / \mathrm{mL})$ & 10 & $28.9(118 \%)$ & 19 & $20 \cdot 6(85 \%)$ \\
\hline \multicolumn{5}{|l|}{ Emtricitabine } \\
\hline$A \cup C_{\text {tau }}(h \cdot n g / m L)$ & 11 & $62900(48 \%)$ & 19 & $11700(17 \%)$ \\
\hline $\mathrm{C}_{\max }(\mathrm{ng} / \mathrm{mL})$ & 12 & $4880(41 \%)$ & 19 & $2060(20 \%)$ \\
\hline $\mathrm{C}_{\mathrm{tau}}(\mathrm{ng} / \mathrm{mL})$ & 10 & $1280(59 \%)$ & 19 & $952(47 \%)$ \\
\hline \multicolumn{5}{|c|}{ Tenofovir alafenamide } \\
\hline$A \cup C_{\text {last }}(h \cdot n g / m L)$ & 12 & $232(53 \%)$ & 19 & $228(47 \%)$ \\
\hline$C_{\max }(\mathrm{ng} / \mathrm{mL})$ & 12 & $246(75 \%)$ & 19 & $233(65 \%)$ \\
\hline \multicolumn{5}{|l|}{ Tenofovir } \\
\hline$A \cup C_{\text {tau }}(h \cdot n g / m L)$ & 10 & $8720(39 \%)$ & 19 & $326(15 \%)$ \\
\hline$C_{\max }(n g / m L)$ & 12 & $443(41 \%)$ & 19 & $182(12 \%)$ \\
\hline $\mathrm{C}_{\text {tau }}(\mathrm{ng} / \mathrm{mL})$ & 10 & $265(73 \%)$ & 19 & $114(18 \%)$ \\
\hline \multicolumn{5}{|c|}{$\begin{array}{l}\text { Data obtained from participants in this study with end-stage renal disease or healthy renal function in historical } \\
\text { controls from intensive pharmacokinetic analysis in a phase } 2 \text { trial with HIV-infected adults. }{ }^{29} A U C_{\text {tau }}=\text { area under the } \\
\text { concentration versus time curve over the dosing interval. } C_{\max }=\text { maximum observed concentration of drug. } \\
C_{\text {tau }}=\text { observed drug concentration at the end of the dosing interval. } T_{\max }=\text { time (observed time point) of } C_{\max } \cdot t_{1 / 2}=\text { estimate } \\
\text { of the terminal elimination half-life of the drug, calculated by dividing the natural log of } 2 \text { by the terminal elimination } \\
\text { rate constant }\left(\lambda_{2}\right) . A U C_{\text {tau }}=\text { area under the concentration versus time curve from time } 0 \text { to the last measureable } \\
\text { concentration. }\end{array}$} \\
\hline
\end{tabular}

Table 3: Steady-state plasma pharmacokinetic parameters in adults with end-stage renal disease or healthy renal function

the most common of which were nausea, hyperkalaemia, and pneumonia. Nausea and hyperkalaemia are common in patients undergoing chronic haemodialysis, and no instances of pneumonia were considered related to study drug. Overall, the adverse event profile observed was consistent with that expected for this study population, and the types and frequencies of adverse events reported in the current study were consistent with those reported in the product information for the elvitegravir, cobicistat, emtricitabine, and tenofovir alafenamide single-tablet regimen. ${ }^{27}$ Because emtricitabine is renally eliminated and its exposures had previously been observed to be increased in adults with mild-to-moderate renal impairment, ${ }^{26}$ analysis of adverse events potentially associated with emtricitabine (ie, among those listed in the prescribing information as having at least $10 \%$ incidence), was carried out separately. The overall incidence of these prespecified events occurred in nearly half of study participants; however, those events considered related to study drug were reported for less than $10 \%$, all were grade 1 or 2 in severity, and none led to premature discontinuation of study drug. The high overall incidence was again not unexpected in our population. These 
Elvitegravir, cobicistat, emtricitabine, and tenofovir alafenamide $(n=55)$

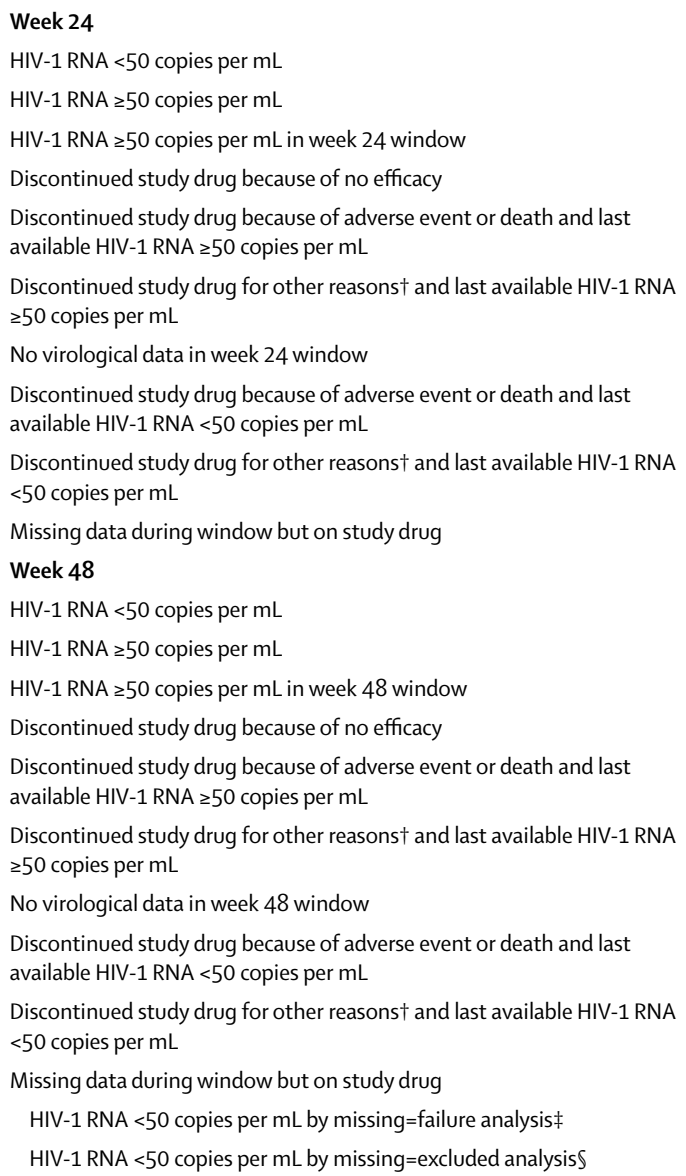

$48(87 \cdot 3 \%, 75 \cdot 5-94 \cdot 7)^{*}$
$1(1 \cdot 8 \%, 0-9 \cdot 7)^{*}$
0
$1(1 \cdot 8 \%)$
0

0

$6(10 \cdot 9 \%)$

$2(3 \cdot 6 \%)$

$4(7 \cdot 3 \%)$

$45(81 \cdot 8 \%, 69 \cdot 1-90 \cdot 9)^{*}$
$2(3.6 \%, 0 \cdot 4-12 \cdot 5)^{*}$
$1(1 \cdot 8 \%)$
$1(1 \cdot 8 \%)$
0
0
$8(14 \cdot 5 \%)$
$2(3 \cdot 6 \%)$
$5(9 \cdot 1 \%)$
$1(1 \cdot 8 \%)$
$46 / 55(83 \cdot 6 \%, 71 \cdot 2-92 \cdot 2)^{*}$
$46 / 47(97 \cdot 9 \%, 88 \cdot 7-99 \cdot 9)^{*}$

Data are $\mathrm{n}(\%, 95 \% \mathrm{Cl})$ or $\mathrm{n}(\%) .{ }^{*} 95 \% \mathrm{Cl}$ s were obtained using the exact method. †Other reasons included participants who discontinued study drug because of investigator's discretion, participant's decision, loss to follow-up, non-compliance with study drug, protocol violation, pregnancy, or study terminated by sponsor. $\ddagger$ The denominato for percentages was the number of participants in the full analysis set. SThe denominator for percentages was the number of participants in the full analysis set with non-missing HIV-1 RNA at each visit.

Table 4: Virological outcomes at weeks 24 and 48

observations support the safety of emtricitabine at a dose of $200 \mathrm{mg}$ daily in the single-tablet regimen of elvitegravir, cobicistat, emtricitabine, and tenofovir alafenamide without dose adjustment in adults with minimal residual renal function undergoing haemodialysis. Study drugrelated adverse events were reported for few participants overall, despite the fact that participants were switched in an unmasked manner to a new combination.

Exposures of tenofovir alafenamide and cobicistat in adults on haemodialysis in our study were consistent with historical data in HIV-infected individuals with healthy renal function. Although the mean steady-state plasma exposures of elvitegravir in participants on haemodialysis in our study were numerically lower than in those with normal renal function in previous studies, these exposures were within the safe and efficacious ranges of other phase 3 trials in HIV-1-infected adults with healthy renal function, and are supported by the safety and efficacy results of this study. Although the plasma concentrations of tenofovir and emtricitabine were increased in the population on haemodialysis compared with historical data in HIV-1-infected adults with healthy renal function and mild-to-moderate renal impairment receiving coformulated elvitegravir, cobicistat, emtricitabine, and tenofovir alafenamide, the overall safety profile was not substantially affected. All participants were expected to have limited or no residual renal function given that they had been on haemodialysis for extended periods of time. Moreover, exposures of tenofovir within this study were less than those in adults on haemodialysis who had received tenofovir disoproxil fumarate. ${ }^{30}$

Finally, switching to elvitegravir, cobicistat, emtricitabine, and tenofovir alafenamide maintained high rates of virological suppression, with treatment-emergent resistance in only one participant who retrospectively was found to have pre-existing resistance at baseline. HIV treatment satisfaction was also improved from baseline, reflecting how simplification to a single-tablet regimen offers a once-daily option for individuals on chronic haemodialysis who often have complicated HIV dosing schedules, multiple comorbidities, and a high pill burden.

Our trial population included individuals with end-stage renal disease who were enrolled at international sites where management of this condition might vary. There were also substantial proportions of women and black participants by contrast with other studies of new drugs for initial or switch therapies. ${ }^{24-26,31,32}$ A potential limitation of our small, openlabel, single-arm study (ie, with no control group) in a patient population with multiple comorbidities is the reduced ability to distinguish background and study drugrelated adverse events. However, given the expected high background adverse event rate in this population, the few discontinuations due to study drug-related adverse events was reassuring. We analysed the primary outcome after 48 weeks of treatment and did not detect serious safety signals; however, analysis of follow-up safety data through 96 weeks will be important and is ongoing. We did not assess residual renal function using more specific measures such as $24 \mathrm{~h}$ timed blood and urine urea or creatinine collection peridialysis, and we did not do bone densitometry studies within our single-arm study. Although tenofovir alafenamide in HIV-infected individuals with mild-to-moderate renal impairment has been shown to be safe for bone, this has not been studied in individuals on dialysis and is an important aim for future investigation. In individuals on haemodialysis who are awaiting a transplantation, a regimen containing cobicistat might not be ideal because of the potential drug-drug interactions. Other tenofovir alafenamide-containing regimens should be investigated within this setting. Finally, although tenofovir alafenamide and emtricitabine have low potential 
for mitochondrial toxicity compared with other nucleoside analogues, further follow-up is required to assess longterm safety in this patient population. ${ }^{33,34}$

Results from our study reinforce the large body of evidence showing that tenofovir alafenamide-containing regimens could be used in individuals with varying degrees of renal impairment. Multiple studies in treatment-naive individuals have shown significant differences in renal safety parameters between tenofovir alafenamide and tenofovir disoproxil fumarate, and virologically suppressed adults with normal to moderately impaired renal function (ie, creatinine clearance $\geq 30 \mathrm{~mL} / \mathrm{min}$ ) who switched to tenofovir alafenamide from tenofovir disoproxil fumarate had significant improvements in total and tubular proteinuria. Moreover, large phase 3 studies of regimens containing the emtricitabine and tenofovir alafenamide NTRI backbone have shown no differences in renal or bone safety compared with that of regimens containing abacavir and lamivudine in participants with normal to moderately impaired renal function (ie, creatinine clearance $\geq 50 \mathrm{~mL} / \mathrm{min}) .^{25,31}$

In conclusion, these results support the use of the single-tablet regimen of elvitegravir, cobicistat, emtricitabine, and tenofovir alafenamide once daily for the treatment of HIV-1 infection in adults with end-stage renal disease on chronic haemodialysis with minimal residual renal function. This regimen might provide a tolerable and convenient option for ongoing treatment of HIV-1 infection in this population and could reduce pill burden and the potential for dosing errors.

Contributors

JJEJ, J-DL, RK, JS, AKW, JLS, CM, EC, AW, BS, and MM enrolled participants onto the study, analysed data, independently interpreted the results, and edited and approved this report. DS and MD designed the study. SJ did the data analyses, which were reviewed and interpreted by SC, SRM, AC, MD, and DS. The first draft of the report was written by JJEJ and DS. All authors were involved in the development of the primary manuscript, interpretation of data, contributed to edits of the final report, and read and approved the final version.

\section{Declaration of interests}

JJEJ is an ad hoc consultant to Gilead Sciences, Merck, Janssen, and ViiV Healthcare. Support for JJEJ was received by The University of North Carolina (NC, USA) from Gilead Sciences for this study. The University of North Carolina receives research contracts from Janssen and ViiV Healthcare that support JJEJ for work outside this study. J-DL has received research grants and personal fees for serving on advisory boards from Gilead Sciences. RK reports research grants from Gilead Sciences. JS has received speaker's bureau income from Gilead Sciences, Janssen, and Merck, and research grants from Gilead and ViiV. CM reports receiving research grants from Gilead Sciences, Janssen, Merck, and ViiV, and speaker's bureau income from Gilead Sciences and Merck. AW has received research grants from Gilead Sciences, Janssen, Pfizer, and GlaxoSmithKline, and has served on advisory boards for Gilead Sciences and Janssen. MM has received research grants and income from Gilead Sciences and research grants from ViiV. SC, SRM, SJ, AC, MD, and DSG are employees of Gilead Sciences and hold stock interest in the company. All other authors report no competing interests.

\section{Acknowledgments}

This study was funded by Gilead Sciences. We thank the patients who participated in this trial and their families, the principal investigators (appendix p 40) and their staff, the Gilead Sciences study staff, and Anna Kido (Gilead Sciences, Foster City, CA, USA) for providing editorial assistance.

\section{References}

1 Hsue P, Shreay S, Song X, Meyer N. A longitudinal analysis of comorbidities among human immunodeficiency virus (HIV) patients and matched non-HIV controls in the USA. IDWeek 2016; 26-30 Oct, New Orleans, LA, USA. Abstr 950.

2 Selik RM, Byers RH Jr, Dworkin MS. Trends in diseases reported on US death certificates that mentioned HIV infection, 1987-1999. J Acquir Immune Defic Syndr 2002; 29: 378-87.

3 Wong C, Gange SJ, Moore RD, et al. Multimorbidity among persons living with HIV in the US. Clin Infect Dis 2018; 66: 123-38.

4 Guaraldi G, Orlando G, Zona S, et al. Premature age-related comorbidities among HIV-infected persons compared with the general population. Clin Infect Dis 2011; 53: 1120-26.

5 Abraham AG, Althoff KN, Jing Y, et al. End-stage renal disease among HIV-infected adults in North America. Clin Infect Dis 2015; 60: 941-49.

6 Locke JE, Gustafson S, Mehta S, et al. Survival benefit of kidney transplantation in HIV-infected patients. Ann Surg 2017; 265: 604-08.

7 Locke JE, Mehta S, Sawinski D, et al. Access to kidney transplantation among HIV-infected waitlist candidates. Clin J Am Soc Nephrol 2017; 12: 467-75.

8 Lipford KJ, McPherson L, Hamoda R, et al. Dialysis facility staff perceptions of racial, gender, and age disparities in access to renal transplantation. BMC Nephrol 2018; 19: 5.

9 de Silva TI, Post FA, Griffin MD, Dockrell DH. HIV-1 infection and the kidney: an evolving challenge in HIV medicine. Mayo Clin Proc 2007; 82: 1103-16.

10 Lucas GM, Lau B, Atta MG, Fine DM, Keruly J, Moore RD Chronic kidney disease incidence and progression to end-stage renal disease in HIV-infected individuals: a tale of two cities. J Infect Dis 2008; 197: 1548-57.

11 Trullas JC, Mocroft A, Cofan F, et al. Dialysis and renal transplantation in HIV-infected patients: a European survey. J Acquir Immune Defic Syndr 2010; 55: 582-89.

12 Fernando SK, Finkelstein FO, Moore BA, Weissman S. Prevalence of chronic kidney disease in an urban HIV infected population. Am J Med Sci 2008; 335: 89-94.

13 Lucas GM, Ross MJ, Stock PG, et al. Clinical practice guideline for the management of chronic kidney disease in patients infected with HIV: 2014 update by the HIV Medicine Association of the Infectious Diseases Society of America. Clin Infect Dis 2014; 59: e96-138.

14 Smit M, Brinkman K, Geerlings S, et al. Future challenges for clinical care of an ageing population infected with HIV: a modelling study. Lancet Infect Dis 2015; 15: 810-18.

15 Schwartz EJ, Szczech LA, Ross MJ, Klotman ME, Winston JA Klotman PE. Highly active antiretroviral therapy and the epidemic of HIV+ end-stage renal disease. J Am Soc Nephrol 2005; 16: 2412-20.

16 Gunthard HF, Saag MS, Benson CA, et al. Antiretroviral drugs for treatment and prevention of HIV infection in adults: 2016 recommendations of the International Antiviral Society USA panel. JAMA 2016; 316: 191-210.

17 European AIDS Clinical Society. Guidelines version 9.0. Brussels: European AIDS Clinical Society, published October, 2017. www.eacsociety.org/files/guidelines_9.0-english.pdf (accessed May 30, 2018)

18 US Panel on Antiretroviral Guidelines for Adults and Adolescents. Guidelines for the use of antiretroviral agents in HIV-1-infected adults and adolescents. Washington DC: U S Department of Health and Human Services, March 27, 2018. https://aidsinfo.nih.gov/ contentfiles/lvguidelines/adultandadolescentgl.pdf (accessed May 30, 2018).

19 Izzedine H, Launay-Vacher V, Baumelou A, Deray G. An appraisal of antiretroviral drugs in hemodialysis. Kidney Int 2001; 60: 821-30.

20 Hall AM, Hendry BM, Nitsch D, Connolly JO. Tenofovir-associated kidney toxicity in HIV-infected patients: a review of the evidence. Am J Kidney Dis 2011; 57: 773-80.

21 Sabin CA, Ryom L, d'Arminio Monforte A, et al. Abacavir use and risk of recurrent myocardial infarction. AIDS 2018; 32: 79-88.

22 Boyle SM, Lee DH, Wyatt CM. HIV in the dialysis population: current issues and future directions. Semin Dialysis 2017; 30: 430-37. 
23 Ray AS, Fordyce MW, Hitchcock MJM. Tenofovir alafenamide: a novel prodrug of tenofovir for the treatment of human immunodeficiency virus. Antivir Res 2016; 125: 63-70.

24 Sax PE, Wohl D, Yin MT, et al. Tenofovir alafenamide versus tenofovir disoproxil fumarate, coformulated with elvitegravir, cobicistat, and emtricitabine, for initial treatment of HIV-1 infection: two randomised, double-blind, phase 3, non-inferiority trials. Lancet 2015; 385: 2606-15.

25 Gallant JE, Daar ES, Raffi F, et al. Efficacy and safety of tenofovir alafenamide versus tenofovir disoproxil fumarate given as fixed-dose combinations containing emtricitabine as backbones for treatment of HIV-1 infection in virologically suppressed adults: a randomised, double-blind, active-controlled phase 3 trial. Lancet HIV 2016; 3: e158-65.

26 Pozniak A, Arribas JR, Gathe J, et al. Switching to tenofovir alafenamide, coformulated with elvitegravir, cobicistat, and emtricitabine, in HIV-infected patients with renal impairment: 48 week results from a single-arm, multi-center, open-label, phase 3 study. J Acquir Immune Defic Syndr 2016; 71: 530-37.

27 GENVOYA (elvitegravir, cobicistat, emtricitabine, and tenofovir alafenamide) summary of product characteristics. Cambridge: Gilead Sciences; February, 2018. http://www.ema.europa.eu/docs/ en_GB/document_library/EPAR_-_Product_Information/ human/004042/WC500197861.pdf (accessed May 30, 2018).

28 Smith F, Hammerstorm T, Soon G, et al. A meta-analysis to assess the FDA DAVP's TLOVR algorithm in HIV submissions. Drug Inf J 2011; 45: 291-300.
29 STRIBILD (elvitegravir, cobicistat, emtricitabine, and tenofovir disoproxil fumarate) summary of product characteristics. Cambridge: Gilead Sciences; February, 2018. http://www.ema. europa.eu/docs/en_GB/document_library/EPAR_-_Product Information/human/002574/WC500144272.pdf (accessed May 30, 2018)

30 Viread (tenofovir disoproxil fumarate) summary of product characteristics. Cambridge: Gilead Sciences; Nov, 2013.

31 Gallant J, Lazzarin A, Mills A, et al. Bictegravir, emtricitabine, and tenofovir alafenamide versus dolutegravir, abacavir, and lamivudine for initial treatment of HIV-1 infection (GS-US-380-1489): a double-blind, multicentre, phase 3, randomised controlled non-inferiority trial. Lancet 2017; 390: 2063-72.

32 Walmsley SL, Antela A, Clumeck N, et al. Dolutegravir plus abacavir-lamivudine for the treatment of HIV-1 infection. N Engl J Med 2013; 369: 1807-18.

33 Stray KM, Park Y, Babusis D, et al. Tenofovir alafenamide (TAF) does not deplete mitochondrial DNA in human T-cell lines at intracellular concentrations exceeding clinically relevant drug exposures. Antiviral Res 2017; 140: 116-20.

34 Venhoff N, Setzer B, Melkaoui K, Walker UA. Mitochondrial toxicity of tenofovir, emtricitabine and abacavir alone and in combination with additional nucleoside reverse transcriptase inhibitors. Antivir Ther 2007; 12: 1075-85. 